\title{
Effect of High Gastric Residual Volume on The Critically Ill Patients' Outcomes
}

\author{
Ghada Shalaby Mahran ${ }^{1}$, Mona Aly Mohammed ${ }^{2}$ \& Manal Mohammed Abd-Elneem ${ }^{3}$ \\ 1. Assistant Professor of Critical Care and Emergency Nursing, Faculty of Nursing, Assiut University, Egypt. \\ 2. Assistant Professor of Critical Care and Emergency Nursing, Faculty of Nursing, Assiut University, Egypt. \\ ${ }^{3 .}$ Lecture of Critical Care and Emergency Nursing, Faculty of Nursing, Assiut University, Egypt.
}

\begin{abstract}
Background: Gastrointestinal (GI) dysfunction is a danger for critically sick patients who receive mechanical breathing. During the course of enteral feeding in the early stages of critical illness, gastric residual volume (GRV) is used as a surrogate metric for GI dysfunction. This study aimed to investigate the effect of high gastric residual volume on critically ill patient outcomes. Design: A prospective, observational research design. Methods: A purposive sample of 60 adult critically ill patients of both sex from the intensive care units (sICU) at Assiut University Hospital, Egypt were included in the study from August 2020 to April 2021. According to the measurement of gastric residual volume, those patients were not randomly allocated in group 1[the normal limit of gastric residual volume (NGRV) <500] and group 2 [High gastric residual volume (HGRV) >500]. Five tools were used to collect data included patient's assessment tool, APACHE 11 score, nutritional intervention assessment tool, gastric residual volume assessment tool, and Patient outcomes assessment tools. Results: there was a significant decrease in the Length of ICU stay, duration of mechanical ventilation, and mortality rate in NGRV group than the HGRV group (11.04 \pm 4.12 versus $13.91 \pm 5.88,8.43 \pm 4.5$ versus $13.02 \pm 5.04,30.4 \%$ versus $70.3 \%$ ) respectively. Conclusion: HGRV had an adverse effect on critically ill patients' outcomes such as high ICU mortality, long duration of mechanical ventilation and long ICU stay. Recommendation: Frequent monitoring of gastric residual volume and gastric residual volume protocol and guidelines should be applied in ICU.
\end{abstract}

\section{Keywords: Enteral feeding, Gastrointestinal dysfunction, Gastric residual volume \& Mechanical ventilation.}

\section{Introduction:}

Enteral feeding (EF) via enteral tubes is administered to patients who have a functional and accessible gastrointestinal (GI) tract but are unable to consume or absorb enough foods to maintain adequate nutrition and hydration. Enteral tubes can be placed into the patient's GI tract in intensive care units through the nose, mouth, stomach, or small intestine to provide liquid nutrients, fluids, and medications. (Lord, 2018). The tubes can be broad or small, and they can be gastric or intestinal in nature. They can be placed in the nose or in the oropharynx. Although enteral feeding tubes are advantageous, there are hazards associated with their usage, as with most medical procedures (Farsi et al., 2020).

The risk of delayed gastric emptying (DGE) is increased in patients with enteral tubes who are critically ill. Half of all adults who use mechanical ventilation (MV) suffer from DGE (Nguyen et al., 2008). DGE can cause a continuous feeling of fullness, an increase in gastric residual volume (GRV), nausea, and vomiting, as well as GI changes as abdominal distension, constipation, diarrhea, increased abdominal circumference, and subjective discomfort, all of which can negatively impact nutritional status and increase the risk of aspiration, gastroesophageal reflux, pneumonia, and length of stay in the hospital (Chen et al 2013; Reignier et al., 2013; Stewart, 2014; Guo, 2015; Nasiri, et al, 2017; \& Poveda et al, 2018).

A high GRV is regarded to be a predictor of GI motility issues in general, as well as delayed stomach emptying in particular. In recent years, critical care and nutrition recommendations have advocated monitoring GRV and delaying or stopping enteral nutrition (EN) if GRV is too high or too low. It's also one of the most extensively used and well-known nursing approaches in the ICU to assess the feeding tolerance, prevent gastric emptying delay and intolerance which may lead to adverse effect on ICU patients outcomes (Elke et al, 2015; \& Brasiel et al, 2020)

Gastric residual volume (GRV) of 150 to $500 \mathrm{~mL}$ on two occasions at two consecutive measures, or a GRV of $>500 \mathrm{~mL}$, or the presence of vomiting were all considered indicators of GI intolerance. When GRVs are high, nurses frequently withhold or reduce the hourly enteral nutrition administration rate, although the GRV threshold is unknown (Guo, 2015).

Nevertheless, to do so in patients with high gastric residual volume (HGRV) results in a reduction in the 
amount of food consumed, which could indicate an energy deficit that is harmful (Montejo et al., 2010). Current recommendations, which define the international guidelines for parenteral and enteral nutrition, emphasize the need of applying strategies such as post-pyloric continuous nutrition and the use of prokinetics when maintaining a high GRV (more than 500mL) ( Bankhead et al., 2009 \& Brasiel et al., 2020)

\section{Significance of the study}

The prevalence of feeding intolerance varied significantly (range: 2\%-75\%), with a pooled proportion of $38.3 \%$ (Blaser, et al., 2014). Previous studies have demonstrated delayed stomach emptying in over half of mechanically ventilated patients and up to $85 \%$ in some diagnostic categories, such as individuals with polytrauma, traumatic brain injury, and sepsis (Gungabissoon et al., 2014). At Assiut university hospital, the medical records of the general intensive care unit patients indicated that the incidence of gastrointestinal dysfunction during the year of 2019 was $54 \%$ (11\% elevated gastric residual volume only) with $50 \%$ mortality.

\section{Operational definitions}

Patient outcomes: Patient outcomes include gastrointestinal complications, duration of mechanical ventilation, length of ICU stay, and mortality.

Aim of the study

The aim of this study was to: Investigate the effect of high gastric residual volume on critically ill patient outcomes

\section{Research question:}

What is the effect of high gastric residual volume on critically ill patient outcomes?

\section{Patients and Methods}

Design: A prospective, observational research design was used.

Setting: The research was carried out in Egypt's Assiut University Hospital's intensive care units. These units include: General ICU (16 beds in four different rooms, nurse patient ratio 1:3), trauma ICU (16 beds in three separate rooms, nurse patient ratio 2:3), and anesthetic ICU (12 beds in three separated rooms, nurse patient ratio 1:2). In terms of equipment, amenities, and paternity leave, all ICUs were the same.

Subject: A purposive sample of 60 adult critically ill patients of both sexes was enrolled in the study from August 2020 to April 2021. According to the measurement of their GRV, those patients were not randomly assigned to two groups [group 1: normal limit of GRV (NGRV) <500] and [group 2: high GRV $(H G R V) \geq 500]$.
Inclusion criteria: Adult male and female patients (18-60 years old), on mechanical ventilation who received early enteral feeding (within 24-48 hours) and had an indication for EN for at least 5 days.

Exclusion criteria: Patients who have had abdominal surgery and have experienced gastrointestinal bleeding, acute pancreatitis, esophageal reflux, and pregnancy.

Calculation of sample size:

$$
n=\frac{\mathrm{np}(1-\mathrm{p})}{\mathrm{n}-1\left(d^{2} \div \mathrm{z}^{2}\right)+\mathrm{p}(1-\mathrm{p})}
$$

$\mathrm{n}=$ sample size

$\mathrm{z}=$ level of confidence according to the standard normal distribution (for a level of confidence of $95 \%$, $\mathrm{z}=1.96)$

$\mathrm{p}=$ estimated proportion of the population that presents the characteristic of the study sample (when unknown we use $\mathrm{p}=0.5$ )

$\mathrm{d}=$ tolerated margin of error (for example we want to know the real proportion within $5 \%$ ) $=0.05$

Tools: Five tools were used to collect data.

Tool (1): Patient's assessment tool:

This tool was developed by the researcher after reviewing of literature (Brasiel et al., 2020) to form base line data for the patients. This tool composed of one part ( Patient demographic and clinical data)

It included demographic data (age, sex), clinical data which included (patient diagnosis, date of intensive care unit admission and discharge)

Tool (2): APACHE 11 score (acute physiology and chronic health evaluation)

The APACHE-II scoring system is used to assess the severity of disease for adult patients admitted to intensive care units. There are three parts to the APACHE-II score. The APACHE-II score's first component (biggest component) is generated from 12 clinical measurements taken within 24 hours of admission to the ICU. The variables are internal temperature, heart rate, mean arterial pressure, respiratory rate, oxygenation, arterial $\mathrm{pH}$, serum sodium, serum potassium, serum creatinin, haematocrit, white blood cells count and Glasgow coma scale. The second component is age adjustment, which adds one to six points for patients over 44 years old. Chronic health evaluation is the APACHEthird II's component. An additional adjustment is made for patients with severe and chronic organ failure involving the heart, lungs, kidneys, liver and immune system. (Naved et al., 2011).

Tool (3): Nutritional Intervention Assessment Tool The researcher developed this tool after conducting a literature review (Farsi et al., 2020) to determine the appropriate feeding volume for patients. This tool composed of four parts. 
Part one: determining the correct position of the nasogastric tube.

Part two: Evaluation of the feeding formula chosen (as intact formula, elemental formula, renal formula, and volume limited formula

Part three: Assessment of the amount of food that has been delivered.

Part four: the Harris-Benedict equation was used to compute the total number of calories and the required volume per patient

Tool 4: Gastric residual volume assessment tool: This tool was developed by the researcher after reviewing of literature (Kar et al., 2015) to assess gastric residual volume. This tool composed of two parts:

Part one: Assessment of gastric residual Amount.

Part two: Assessment of gastric residual volume intervention.

Tool 5: Patient outcomes assessment tools

After analyzing the literature (Brasiel et al., 2020), the researcher designed this instrument to assess patient outcomes. There are four parts in this tool.

Part one: Assessment of gastrointestinal complication as diarrhea, constipation, distention, and emesis.

Part two: Assessment of MV duration.

Part three: Assessment of length of ICU stay.

Part four: Assessment of mortality rates.

Method

Data collecting technique: the research was carried out in three stages.

Preparatory phase: The phase involved:-

- After explaining the study's purpose to the competent authorities at Assiut University's general, anesthetic, and trauma intensive care unit, official and non-official authorization to conduct the study was obtained.

Pilot study:

- A six-patient pilot study (10 percent of the sample) was conducted to assess the tools' applicability.

- A panel of five specialists was tasked with evaluating the tools' content validity. The scale content validity index for each item in the sheet was 0.94 .

- The reliability was done on tool to conduct the study by using Cronbach's alpha the result was 0.90

Ethical consideration:

The research was authorized by the faculty of nursing's ethical committee. Patients or their legal guardians gave their informed permission. During the implementation of the research, there was no risk to the study subjects. The investigation was conducted in accordance with standard clinical research ethics. After discussing the nature and goal of the study, one of the close relatives (father, mother, husband, or wife) gave informed consent. Confidentiality was assured.

Implementation phase:

- Demographic information (age, gender), as well as clinical information (patient diagnosis, date of ICU admission and discharge) were recorded.

- The length of time that mechanical ventilation was used was measured.

- At the time of admission to the ICU, an acute physiologic and chronic health evaluation (APACHE II) was performed.

Nutritional Intervention Assessment:

- Assessment of the correct position of the nasogastric tube.

- The correct location of the nasogastric tube was checked by infusing $50 \mathrm{~mL}$ of air into the tube with a syringe and auscultating the epigastric area, or, if necessary, by radiograph.

- The researchers measured the exposed portion of the tube and compared the length to earlier measurements to ensure the tube was in the correct position.

- Patients were fed in a semi-recumbent position, with the patient's position and tube length remaining constant throughout the study.

The feeding protocol:

- Within 24-48 hours, all patients in both groups began enteral feeding.

- The critical care team calculated and applied a standard blenderized polymeric recipe.

- The identical technique for achieving the EN target was employed in the two groups, starting with bolus EN administration at $25 \mathrm{~mL} / \mathrm{h}$ and rising by $25 \mathrm{~mL} / \mathrm{h}$ every 6 hours until the goal of $85 \mathrm{~mL} / \mathrm{h}$ was reached. EN was reduced to the previously well-tolerated rate and erythromycin was started in the event of EN intolerance, defined as GRV >250 $\mathrm{mL}$ and/or vomiting or only vomiting.

- Patients were bolus fed, with the head of the bed elevated 45 degrees and infusions administered using an open delivery system (the open method uses either a catheter or a syringe).

- After bolus feeding, the feeding tube was flushed with $50 \mathrm{~mL}$ water, and the gastric residual volume was measured.

- Patient positioned in bed semi fowler's (head of bed 45-60 degrees) as tolerated.

- Tube was flushed with $30 \mathrm{ml}$ water after the complete residual volume was obtained.

Assessment of the total number of calories

- The Harris-Benedict equation, which takes into account the gender, body weight, height, age, and activity level of the patient to determine the most 
appropriate volume, was used to compute the total number of calories and the required volume per patient.

- Calculation of $24 \mathrm{hrs}$ energy requirements as following:

Total energy expenditure (requirements) =basal metabolic rate $(\mathrm{BMR}) \times($ stress factor + activity factor+food thermic effect).

- BMR was calculated by Harris-Benedict equation: Male $($ Kcal-D) $=66.5+(13.75 \times$ weight $\mathrm{kg})+(5-$ $\times$ height $\mathrm{cm})-(6.775 \times$ age $\mathrm{y})$.

Female $($ Kcal-D) $=655.1+(9.563 \times$ weight $\mathrm{kg})+$ (1.85×height $\mathrm{cm})-(4.67 \times$ age $y)$.

\section{While weight kg was:}

- Current body weight (CBW) in normal weight subjects.

- Adujusted body weight $\{\mathrm{ABW}=(\mathrm{CBW}+\mathrm{IBW})$ $\div 2\}$ in obese and underweight subjects.

- A stress factor and activity factor will be estimated based on the patient's condition

- As the participants were sedentary (little or no exercise), the patients' BMR was multiplied by 1.2 to calculate the total calories

Gastric residual volume assessment and intervention.

- The GRV was measured every 6 hours on the first EN day, every 8 hours on the second EN day, and on a daily basis after the third day of tolerated EN.

- The GRV was measured using one of two methods: (1) gravity drainage using a stomach tube connected to a drainage bag for 10 minutes, or (2) syringe aspiration (using a 50-ml syringe) through the gastric tube.

- The GRV measurements were taken in the patient's current position; no attempt was made to manipulate this variable.

- During the first three days of EN, all patients were given metoclopramide (10 $\mathrm{mg}$ every 8 hours) intravenously as a preventive prokinetic drug.

- Before determining the GRV, the feeding was stopped for 30 minutes. If the residual volume was greater than $500 \mathrm{~mL}$ or between, $200,500 \mathrm{~mL}$, and the patients had abdominal distension, no bowel sounds, or nausea or vomiting, the nurses stopped the enteral tube feed after the measurement.

- If the GRV was less than $200 \mathrm{~mL}$ and there was a low risk of aspiration, feeding was promptly resumed at the original pace.

- Patients were monitored on a daily basis to see if they had any gastrointestinal issues.

- Gastrointestinal complications were defined as follows:
1.Tympany and/or absence of bowel noises on daily physical examination indicate abdominal distention.

2.High gastric residuals: The gastric residual was termed high if the recovered volume was equal to or greater than $500 \mathrm{ml}$.

3.Vomiting: the ejection of enteral formula through the mouth.

4.Diet regurgitation: the presence of enteral formula in the oral or nasal cavities, with or without externalization.

5.Diarrhea: five or more liquid stools in a 24-h period or an estimated stool volume equal or greater than $2,000 \mathrm{ml} /$ day.

Patients were followed until the end of EN.

Evaluation phase:

Gastrointestinal problems (vomiting, distention, diarrhea, and constipation), length of hospital stay, duration of mechanical ventilation, and death were all examined in the two groups.

\section{Statistical analysis:}

- Data were computerized and analyzed by computer programmed SPSS (ver.16). Quantitative data were compared using independent samples t-test for comparing two groups. Qualitative variables were compared using

- Chi-square test to determine Significance.

- Descriptive data are reported as mean \pm SD and frequency distributions $(\mathrm{n}, \%)$ for categorical variables.

- The critical value of the tests "P" was considered statistically significant when $\mathrm{p}$.value $<0.05$.

- Correlation between multiple variables was done by using curve estimation. 
Result:

Table (1): Frequency distribution of patients regarding demographic characteristics and clinical data at admission $(\mathbf{n}=60)$ :

\begin{tabular}{|c|c|c|c|c|}
\hline & Item & $\begin{array}{c}\text { Normal limit of } \\
\text { GRV(NGRV) } \\
(n=23)\end{array}$ & $\begin{array}{r}\text { HGRV } \\
(\mathbf{n}=37)\end{array}$ & P-value \\
\hline \multicolumn{2}{|l|}{ Age } & $42.08 \pm 14.86$ & $45.70 \pm 15.44$ & 0.37 \\
\hline \multicolumn{2}{|c|}{ APACHE 11 score } & $13.62 \pm 6.62$ & $16.51 \pm 7.36$ & 0.116 \\
\hline \multirow{2}{*}{ Sex } & Male & $19(82.6 \%)$ & $29(78.4 \%)$ & \multirow{2}{*}{.69} \\
\hline & Female & $4(17.4 \%)$ & $8(21.6 \%)$ & \\
\hline \multirow{6}{*}{ Diagnosis } & COPD & $4(17.4 \%)$ & $8(21.6 \%)$ & \multirow{6}{*}{.42} \\
\hline & Septic shock & $4(17.4 \%)$ & $6(16.21 \%)$ & \\
\hline & Traumatic brain injury & $5(21.7 \%)$ & $9(24.3 \%)$ & \\
\hline & Chest trauma & $4(17.4 \%)$ & $7(18.91 \%)$ & \\
\hline & Renal failure & $3(13.04 \%)$ & $4(10.81 \%)$ & \\
\hline & Pneumonia & $3(13.04 \%)$ & $3(8.10 \%)$ & \\
\hline
\end{tabular}

*Significant difference $p$.value $<0.05$.

-Independent samples $t$-test for comparing two groups

- Chi-square test for qualitative variables.

Table (2): Comparison between the two groups in relation to selected feeding formula during the period of elevated GRV

\begin{tabular}{|c|c|c|c|c|}
\hline & Item & $\begin{array}{c}\text { Normal limit of } \\
\text { GRV }(n=23)\end{array}$ & $\begin{array}{l}\text { HGRV } \\
(n=37)\end{array}$ & P-value \\
\hline \multirow[t]{6}{*}{ selected formula } & Standard intact formula & $13(56.5 \%)$ & $15(40.5 \%)$ & \multirow{6}{*}{.115} \\
\hline & elemental formula & $5(21.7 \%)$ & $19(51.4 \%)$ & \\
\hline & Renal formula & $1(4.3 \%)$ & $2(5.4 \%)$ & \\
\hline & volume restricted formula & $2(8.7 \%)$ & $0 .(0 \%)$ & \\
\hline & volume restricted, ,elemental formula & $1(4.3 \%)$ & $1(2.7 \%)$ & \\
\hline & disease specific formula & $1(4.3 \%)$ & $0 .(0 \%)$ & \\
\hline
\end{tabular}

* Significant difference $p$.value $<0.05 . \quad$ - Chi-square test for qualitative variables.

Table (3): Comparison between the two groups in relation to feeding amount per day and Total amount of GRV, during the period of elevated GRV $(n=60)$ :

\begin{tabular}{|l|l|l|l|}
\hline \multicolumn{1}{|c|}{ Item } & \multicolumn{1}{|c|}{$\begin{array}{c}\text { Normal limit of } \\
\text { GRV(n=23) }\end{array}$} & $\begin{array}{c}\text { HGRV } \\
(\mathbf{n = 3 7 )}\end{array}$ & P-value \\
\hline feeding amount per day & $1925.43 \pm 1038.40$ & $1840.89 \pm 676.48$ & .70 \\
\hline Total amount of GRV & $326.95 \pm 147.96$ & $947.29 \pm 300.68$ & $0.001^{*}$ \\
\hline
\end{tabular}

* Significant difference $p$.value $<0.05$ - Independent samples t-test for comparing two groups

Table (4): Comparison between the two groups in relation to gastrointestinal complication during the period of elevated GRV $(n=60)$ :

\begin{tabular}{|l|c|c|c|}
\hline \multicolumn{1}{|c|}{ Item } & $\begin{array}{c}\text { Normal limit of } \\
\text { GRV(n=23) }\end{array}$ & $\begin{array}{c}\text { HGRV } \\
(\mathbf{n}=37)\end{array}$ & P-value \\
\hline Diarrhea & $6(26.1 \%)$ & $7(18.9 \%)$ & .51 \\
\hline Constipation & $5(21.7 \%)$ & $18(48.6 \%)$ & $.03^{*}$ \\
\hline Vomiting & $2(8.7 \%)$ & $14(37.8 \%)$ & $.01^{*}$ \\
\hline Distention & $4(17.4 \%)$ & $20(54.1 \%)$ & $.005^{*}$ \\
\hline
\end{tabular}

* Significant difference $p$.value $<0.05 . \quad$-Chi-square test for qualitative variables.

Table (5): Comparison between the two groups in relation to patients out comes (Length of ICU stay, Duration of connection with mechanical ventilation, and mortality):

\begin{tabular}{|l|l|l|l|}
\hline \multicolumn{1}{|c|}{ Item } & \multicolumn{1}{|c|}{$\begin{array}{c}\text { Normal limit of } \\
\text { GRV(n=21) }\end{array}$} & \multicolumn{1}{|c|}{$\begin{array}{c}\text { HGRV } \\
(\mathbf{n = 3 7})\end{array}$} & P-value \\
\hline Length of ICU stay & $11.04 \pm 4.12$ & $13.91 \pm 5.88$ & $0.04^{*}$ \\
\hline mechanical ventilation duration & $8.43 \pm 4.5$ & $13.02 \pm 5.04$ & $.001^{*}$ \\
\hline Mortality & $7(30.4 \%)$ & $26(70.3 \%)$ & $.006^{*}$ \\
\hline
\end{tabular}

* Significant difference $p$.value $<0.05$.

-Independent samples t-test for comparing two groups

-Chi-square test for qualitative variables. 
Table (1): Shows frequency distribution of patients in the two groups regarding demographic characteristics and clinical data at admission: Regarding to age the results of the current study revealed that the mean and SD of patients' age in NGRV and HGRV were $(42.08 \pm 14.86$ and $45.70 \pm 15.44)$ respectively. Regarding to APACHE 11 score the results of the current study revealed that the mean and SD of patients' APACHE 11 score in NGRV and HGRV were (13.62 \pm 6.62 and 16.517.36) respectively. Regarding to sex, it was noticed that a highly percent of patients in NGRV and HGRV were male $(82.6 \%)$ and $(78.4 \%)$ respectively. Regarding to diagnosis, results revealed a relatively high percentage of patients in NGRV and HGRV were traumatic brain injury 5 (21.7\%), 9 (24.3\%) respectively and there was no statistically Significant difference between the two groups in all parameters (p. value $>0.05$ ).

Table (2): Shows comparison between the two groups in relation to selected feeding formula during the period of elevated GRV:

Concerning with selected feeding formula, most of cases in NGRV group received Standard intact formula $(56.5 \%)$ in the other hand $(51.4 \%)$ from the HGRV group received elemental formula. There was statistically Significant difference between the two groups (p. value $<0.05$ ).

Table (3): Shows Comparison between the two groups in relation to feeding amount per day and total amount of GRV, during the period of elevated GRV. In concern delivered feeding amount, the current study revealed that the mean and SD of patients' delivered feeding amount in NGRV and HGRV were $(1925.43 \pm 1038.40$ and $1840.89 \pm 676.484)$ respectively. Regarding to Total amount of GRV, the current study revealed that the mean and SD of patients' total amount of GRV in NGRV and HGRV were (326.95 \pm 147.96 and 947.29 $\pm 300.68)$ respectively. There was statistically Significant difference between the two groups (p. value $<0.05$ ) regarding total amount of GRV.

Table (4): Shows comparison between the two groups in relation to gastrointestinal complication during the period of elevated GRV: Concerning with gastrointestinal complication, diarrhea occurred in $26.1 \%$ of patients in NGRV group versus $18.9 \%$ of patients in the HGRV group. Constipation occurred in $21.7 \%$ of patients in NGRV group versus $48.6 \%$ of patients in the HGRV group. Vomiting occurred in $8.7 \%$ of patients in NGRV group versus $37.8 \%$ of patients in the HGRV group. Distension occurred in $17.4 \%$ of patients in NGRV group versus $54.1 \%$ of patients in the HGRV group. There was statistically considerable difference between the two groups (p. value $<0.05)$ in all GIT complication except diarrhea.
Table (5): Shows comparison between the two groups in relation to patients out comes (Length of ICU stay, duration of connection with mechanical ventilation, and mortality): Concerning to Length of ICU stay, there was obvious decrease in the Length of ICU stay in NGRV group than the HGRV group $11.04 \pm 4.12$ versus $13.91 \pm 5.88$ respectively. Concerning with duration of mechanical ventilation, there was dramatic decrease in NGRV group than the HGRV group $8.43 \pm 4.5$ versus $13.02 \pm 5.04$ respectively. Concerning to mortality rate, there was a highly considerable increase in the mortality rate in NGRV group than the HGRV group 7 (30.4\%) versus $26(70.3 \%)$ respectively. There was statistically Significant difference between the two groups in all outcomes (p. value <0.05).

\section{Discussion}

With new research pointing to nutritional therapy as a means of modifying the underlying illness process and improving patient outcomes, it was noticed that much of the recent focus on enteral nutrition has been on improving its delivery (Guo, 2015).

In terms of sex and diagnosis, both groups had mostly male participants with traumatic brain injury, with no statistically significant difference between the two groups. This result was consistent with previous research (Soroush et al., 2018 \& Farsi et al., 2020). However, because this is characteristic of the overall TBI population, the higher male-to-female ratio and the young age of the participants are comprehensible. This is due to the fact that males are more prone than females to get head injuries when driving (the most prevalent cause of head injury). Furthermore, following a head injury, patients with high intracranial pressure have been observed to have delayed GE, and high intracranial pressure is thought to be the major cause of impaired stomach motility and emptying. The findings of this investigation demonstrated that the two groups of patients had similar diagnoses and demographic features. These findings were in agreement with (Wong et al., 2011) study which also showed no statistically difference between the two groups regarding diagnosis of study sample.

Semi-elemental formulations contain hydrolyzed macronutrients (partially or fully) to aid digestion and absorption of nutrients (Escuro, Hummell, 2016 \& Harvey, et al., 2017). Patients with a clogged GI tract will most likely benefit from these products. In terms of feeding formula, the majority of cases in the NGRV group received Standard intact formula, while those in the HGRV group received elemental formula. In terms of delivered feeding amount, the current study found that HGRV delivered feeding amount was lower than NGRV delivered feeding amount. 
This can be explained by the fact that nursing care for patients with high GRVs differs; some institutions elected to start prokinetics and continue feedings at the same or reduced rates, while others stopped feeding and reassessed the situation. After a scenario of high gastric residual volume (HGRV) is recognized, holding enteral feeding is a common technique. However, in certain patients, doing so results in a decrease in the amount of food they receive, which could suggest a dangerous energy deficit. These findings echoed those of (Elke et al., 2015) who mentioned that Patients having a high GRV consumed fewer calories and vomited more frequently than those with a normal GRV. Mentec et al., 2001 mentioned that high GAV was associated with a lower feed intake and vomiting.

In terms of gastrointestinal complications, the HGRV group had a significant increase in vomiting and distension compared to the NGRV group ( $\mathrm{p}$. value 0.05). This is due to the fact that higher stomach content might cause vomiting. As a result, greater stomach residual volume is undesirable in enteral feeding patients. These findings were supported by (Bonten, 2011). While Montejo et al., 2010. compared the effects of $200 \mathrm{~mL}$ (control group) versus $500 \mathrm{~mL}$ GRV (study group) in 28 Spanish ICUs in an open, prospective RCT. Although the difference appeared to be related entirely to the prevalence of high GRV, the control group had a higher rate of GI issues. Other specified GI problems such as abdominal distension, vomiting, food regurgitation, or diarrhea did not differ substantially between groups.

When compared to patients who do not have upper digestive intolerance, intolerant patients receive less meal volume and have a worse prognosis in terms of ICU duration and mortality (Montejo et al., 2010). The NGRV group had a considerably shorter length of ICU stay, duration of mechanical ventilation, and mortality rate than the HGRV group in terms of patient outcomes. According to Mentec et al., 2001 high GRV was discovered as an early indicator of upper intestine intolerance. Even after adjusting for Simplified Acute Physiology Score II (OR, 1.48; 95 percent CI, 1.04-2.10; p =.028), upper digestive intolerance was related with a longer ICU stay and a greater ICU mortality (Mentec et al., 2001). Previous studies have shown that the number of GI symptoms experienced in the ICU over a single day is linked to an increased risk of death (Reintam et al., 2013, \& Reintam et al., 2009). Furthermore, Soroksky et al. (2010) discovered that low GRV (less than $500 \mathrm{ml}$ ) and high GRV (at least one measurement of GRV > $500 \mathrm{ml}$ ) patients had similar lengths of ICU stay, durations of mechanical breathing, and ICU and hospital mortality.

\section{Conclusion}

In ICU patients receiving nasogastric tube feeding, high gastric aspirate volume was prevalent and occurred early. High gastric aspirate volume was connected to a higher rate of long-term mechanical ventilation, a longer ICU stay, and a higher rate of ICU death as an early sign of upper digestive intolerance.

\section{Recommendation:}

- Further study should be done on large number.

- Frequent monitoring of gastric residual volume is recommended.

- Apply gastric residual volume protocol and guidelines.

\section{References:}

- Bankhead R, Boullata J \& Brantley S (2009): A.S.P.E.N. Board of Directors. Enteral nutrition practice recommendations. JPEN J Parenter Enteral Nutr.;33:122-167.

- Blaser, AR. Starkopf, J. Kirsimagi, U. \& Deane, A. (2014): Definition, prevalence, and outcome of feeding intolerance in intensive care: a systematic review and meta-analysis. Acta Anaesthesiol Scand. ;vol. (58), No. (8), Pp914922.

- Bonten, M.J. (2011): Ventilator-associated pneumonia and the gastropulmonary route of infection: a pendulum. Am. J. Respir. Crit. Care Med. 184 (9) 991-993,

- Brasiel PGA, Melo AST \& Aguiar AS (2020): Does enteral nutrition infusion rate impact on the gastric residual volume of critically Ill Patients? Gastroenterol Hepatol Open Access. 2020;11(2):78-82. DOI: 10.15406/ghoa. 11.00418

- Chen, S., Tzeng, Y., Gau, B., Kuo, P., \& Chen, J. (2013): Effects of prone and supine positioning on gastric residuals in preterm infants: A time series with cross-over study. International Journal of Nursing Studies, 50(11), 1459-1467. https://doi.org/10.1016/j.ijnurstu.2013.02.009

- Elke G; Thomas W. Felbinger; \& Daren K. Heyland (2015): Gastric Residual Volume in Critically Ill Patients: A Dead Marker or Still Alive?. Nutr Clin Pract.;30:59-71

- Escuro A. \& Hummell A (2016): Enteral Formulas in Nutrition Support Practice: Is there a better choice for your patient? Nutr Clin Pract, Vol (31), Pp709-22.

- Farsi Z, Maa'soumeh K, Samantha B \& Armin Z (2020): The Effect of Semi-recumbent and Right Lateral Positions on the Gastric Residual Volume of Mechanically Ventilated, Critically Ill Patients. The Journal of Nursing Research, VOL. 28, NO. 4, AUGUST 2020 
- Gungabissoon U, Hacquoil K, \& Bains C (2014): Prevalence -, risk factors, clinical consequences, and - treatment of enteral feed intolerance during critical illness. JPEN J Parenter Enteral Nutr,2015 May;39(4):441-8.

- Guo B (2015): Gastric residual volume management in critically ill mechanically ventilated patients: A literature review. Proceedings of Singapore Healthcare, Vol. 24(3) 171-180.

- Harvey L, Hudson L, Wessel J, Klang M, \& Martin K (2016): ASPEN Safe Practices for Enteral Nutrition Therapy, Journal of Parenteral and Enteral Nutrition, Pp 1-89

- Montejo J. Miñambres, E. Bordeje, L. Mesejo, A. Acosta, J. Heras, A. Ferre, M. FernandezOrtega, F. Vaquerizo C. \& Manzanedo R. (2010): Gastric residual volume during enteral nutrition in ICU patients: the REGANE study. Intensive Care Med 36:1386-1393.

- Kar P, Karen L., Michael H, Marianne J. Chapman \& Adam M. (2015): Measurement of gastric emptying in the critically ill. Clinical Nutrition 34, 557-564.

- Lord, L. (2018): Enteral access devices: Types, function, care, and challenges. Nutrition in Clinical Practice, 33(1), 16-38. https://doi.org/10.1002/ncp.10019

- Mentec H, Dupont H, Bocchetti M, Cani P, Ponche F \& Bleichner G (2001): Upper digestive intolerance during enteral nutrition in critically ill patients: frequency, risk factors, and complications. Crit Care Med.;29: 1955-1961

- Nasiri, M., Farsi, Z., Ahangari, M., \& Dadgari, F (2017): Comparison of intermittent and bolus enteral feeding methods on enteral feeding intolerance of patients with sepsis: A triple-blind controlled trial in intensive care units. Middle East Journal of Digestive Disease, 9(4), 218-227.

- Naved S., Siddiqui, S., \& Khan, F (2011): APACHE-II Score Correlation with Mortality And Length Of Stay In An Intensive Care Unit. Journal of the College of Physicians and Surgeons Pakistan, 21(1).

- Nguyen NQ, Fraser RJ, Bryant LK, Chapman M \& Holloway RH (2008): Diminished functional association between proximal and distal gastric motility in critically ill patients. Intensive Care Med.; 34:1246-1255.

- Poveda, V., Castilho, A., Nogueira, L., FerrettiRebustini, R., \& Silva, R. (2018): Assessing gastric residual volume: A description of nurses' clinical practice. Revista da Escola de Enfermagem da USP, 52, e03352. https://doi.org/10.1590/S1980220X2017038803352
- Reignier J, Mercier E, \& Gouge AL (2013): Effect of not monitoring residual gastric volume on risk of ventilated-associated pneumonia in adults receiving mechanical ventilation and early enteral feeding: a randomized controlled trial. JAMA; 309: 249-256.

- Reintam A, Parm P, Kitus R, Kern H \& Starkopf J (2009): Gastrointestinal symptoms in intensive care patients. Acta Anaesthesiol Scand;53:318e24

- Reintam A, Poeze M, Malbrain ML, Bj€orck M, Oudemans-van Straaten HM \& Starkopf J (2013): Gastrointestinal symptoms during the first week of intensive care are associated with poor outcome: a prospective multicenter study. Intensive Care Med; 39:899e909.

- Soroksky A, Jonathan L, Elieser K, Eduard MD, Avraham M, Asaf M, Tal Man B \& Yuval L (2010): A Simplified Approach to the Management of Gastric Residual Volumes in Critically Ill Mechanically Ventilated patients: A Pilot Prospective Cohort Study. IMAJ, VOL 12 ; september 2010

- Soroush A, Salar I, Abdi A, Momenfar F, \& Hemmatpour B (2018): Studying the effect of abdominal massage on the gastric residual volume in patients hospitalized in intensive care units, journal of Intensive Care, vol (6), P 47.

- Stewart ML (2014): Interruptions in enteral nutrition delivery in critically ill patients and recommendations for clinical practice. Crit Care Nurse 2014; 34 (4)14-21 quiz 2.

- Wong, K, Lee, D, Lin s, \& Huang H (2011): Impact of disease severity on gastric residual volume in critical patients, World J Gastroenterol. Vl. (17), No (15), Pp2007-2012. 